\title{
44. THE INFLUENCE OF MINOR PLANETS ON THE MOTIONS OF COMETS
}

\author{
K. A. SHTEJNS and I. E. ZAL'KALNE \\ Astronomical Observatory, Latvian State University, Riga, U.S.S.R.
}

\begin{abstract}
It is shown that in a sufficiently small interval there is a uniform distribution of the minimum distances between the orbit of a comet and the orbits of minor planets. It is also shown that there is a uniform distribution of the distance of the planet from the point of closest approach when the comet reaches that point. It is found that $\mathrm{P} /$ Daniel may collide with a microplanet of mass $4.05 \times 10^{8} \mathrm{~g}$ during 100 revolutions. It is possible for one of 60 short-period comets to meet during 100 revolutions a microplanet of mass $9.9 \times 10^{9} \mathrm{~g}$ and radius $8.6 \mathrm{~m}$. The changes in reciprocal semimajor axis of a comet arising from close encounters with minor planets are also studied but found to be insignificant.
\end{abstract}

\section{Approaches between Comets and Minor Planets}

It is clear that very many minor planets remain to be discovered. The general opinion is that there exist minor planets with masses smaller than those of the comets. Such minor planets will be referred to as microplanets, and we shall extrapolate the statistical data of the known minor planets to microplanets with masses down to $10^{8} \mathrm{~g}$. We shall specifically consider the influence of minor planets on the motion of periodic comet Daniel, the orbital elements of which are $\Omega=70^{\circ}, i=20^{\circ}, \omega=7^{\circ}, \varphi=35^{\circ}$, $a=3.6 \mathrm{AU}$ (Belyaev, 1966) and the assumed mass $m_{k}=0.782 \times 10^{-19}$ solar masses. In this paper length is generally expressed in astronomical units, mass in solar masses and time in days. We have determined the minimum distances between the mean orbit of P/Daniel and the orbits of 1735 numbered minor planets (Chebotarev, 1968), the heliocentric velocity $v_{p}$ of each planet, the relative velocity $v_{k}^{\prime}$ of the comet and the angle between the velocity vectors. We found that 13 planets have a minimum distance $d$ of less than 0.1 AU, the actual distances being distributed almost uniformly over the interval $(0.0,0.1)$.

Let us demonstrate the uniformity of the distribution of the minimum distances $d$. In the vicinity of the intersection we may consider the trajectories of both minor planet and comet to be rectilinear. Figure 1 shows that the minimum distances between the comet orbit $K K_{1}$ and the planet orbits are equal to the distance from parallel lines to the plane $Q_{1}$, defined by $O O_{1}$ and $K K_{1}$. The microstructure in $d$ will be between the plane $Q_{1}$ and the parallel plane $Q_{2}$ situated at a small distance. Since the number of orbits between $Q_{1}$ and $Q_{2}$ is proportional to the distance between these planes, the distribution is uniform. There will also be a uniform distribution if the path of the comet is through minor planet trajectories that can be expanded into several pencils of parallel trajectories.

The angle $\theta$ between the planet's velocity $v_{p}$ and the comet's relative velocity $v_{k}^{\prime}$ is concentrated near $90^{\circ}$, this value being determined by the angle of intersection between the comet orbit and the nearly circular orbits of the typical minor planets. 
From the statistics we find that $v_{p}$ is almost uniformly distributed over the interval $(0.008,0.015)$ and $v_{k}^{\prime}$ is almost uniform in the range $(0.003,0.010)$. Because of the small range of their variations we can replace $\theta, v_{p}, v_{k}^{\prime}$ and also $\delta=v_{k}^{\prime} / v_{p}$ by their mean values. We can also demonstrate the uniform distribution of the distance $h$ from the point of closest approach which the planet has covered in unperturbed uniform rectilinear motion (or must cover if $h<0$ ) when the comet reaches that point. Indeed, the time in which the minor planet is within this distance is proportional to the distance. If the comet is at the point of closest approach of the orbits, the planet must be somewhere on the circumference of its orbit, which for a radius of $2.90 \mathrm{AU}$ is $18.22 \mathrm{AU}$, and hence the probability $h / 18.22$ corresponds to the length of $h$.

We need to know the distribution of the number of planets according to their masses $m_{p}$ and have therefore analysed in detail Kuiper's (1958) statistics of absolute magnitudes. From observations of a number of minor planets on a plate he determined the so-called completeness factor, i.e., the possibility of finding all the planets
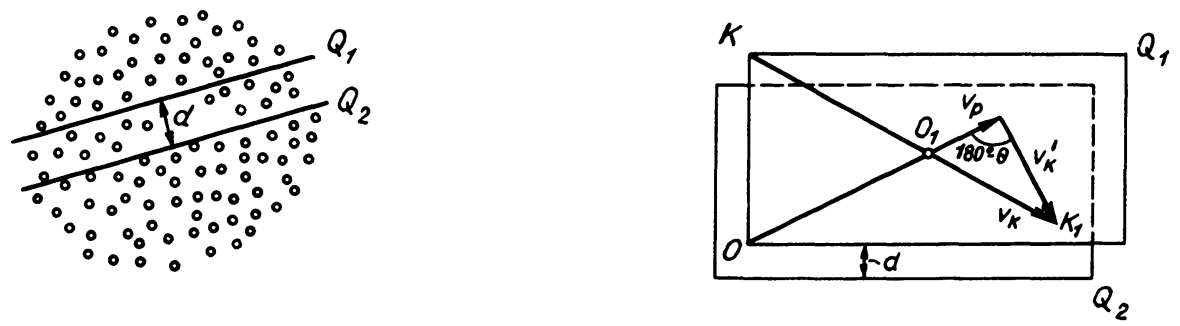

Fig. 1. Demonstration of the uniform distribution of the minimum distances $d$ between the orbit of a comet and the orbits of minor planets.

on the plate and the dependence of the number of minor planets on their magnitudes. On the basis of his data and the assumption that minor planets and microplanets are spheres of density $3.5 \mathrm{~g} \mathrm{~cm}^{-3}$ we have found the relation between the number of minor planets and their mass, namely, that the number $N$ of planets in the interval $\mathrm{d} m_{p}$ is

$$
N \mathrm{~d} m_{p}=\frac{2.3 \times 10^{-14}}{m_{p}^{2.28}} \mathrm{~d} m_{p}
$$

Hence the density of the distribution is

$$
p(N)=\frac{1.28}{m_{p}^{2.28}} \frac{\left(m_{0} M_{0}\right)^{1.28}}{M_{0}^{1.28}-m_{0}^{1.28}},
$$

where $m_{0}$ and $M_{0}$ are the limiting values of the planet masses in the region considered.

\section{Collisions}

A collision between a minor planet and comet takes place if

$$
\rho_{p}+\rho_{k} \geqslant \sqrt{A^{2}+B^{2}}-A,
$$


where $\rho_{p}$ and $\rho_{k}$ are the radii of the planet and the comet (both assumed spherical), and $A$ and $B$ are the semimajor and semiminor axes of the relative hyperbolic orbit of the planet and comet in their mutual sphere of action. If in uniform unperturbed rectilinear motion the minimum distance between the planet and comet is $\Delta_{m}$, then (van Woerkom, 1948)

$$
B^{2}=\Delta_{m}^{2}=d^{2}+h^{2} \sin ^{2} \theta .
$$

We also have

$$
m_{p}=2.5 \times 10^{7} \rho_{p}^{3},
$$

and

$$
A \simeq \frac{k^{2} m_{p}}{v_{k}}
$$

where $k^{2}$ is the gravitational constant. It follows from Equation (3) that

$$
d^{2}+h^{2} \sin ^{2} \theta \leqslant\left(\rho_{p}+\rho_{k}\right)\left(\rho_{p}+\rho_{k}+2 A\right) .
$$

Taking into account the values of $v_{k}^{\prime}$ we have

$$
A<10^{9} \rho_{p}^{3} .
$$

The minor planet of largest mass has $\rho_{p}=2 \times 10^{-6} \mathrm{AU}$; hence

$$
\rho_{p}+10^{9} \rho_{p}^{3}=\rho_{p}\left(1+10^{9} \rho_{p}^{2}\right)<\rho_{p}\left(1+4 \times 10^{-3}\right) \approx \rho_{p},
$$

and the conditions under which collisions take place may be written as

$$
d^{2}+h^{2} \sin ^{2} \theta \leqslant\left(\rho_{p}+\rho_{k}\right)^{2},
$$

i.e., the conditions existing for unperturbed motion.

From these conditions we can calculate the probable number of collisions of the comet with microplanets. The statistics show that 13 of the 1735 numbered minor planets have $d$ in the range $(0.0,0.1)$ and $h$ in $(-9.11,+9.11)$. Since the $d$ and $h$ distributions are uniform and the values themselves independent, the number of microplanets with given $d$ and $h$ is proportional to the area of their variation regions in the $d, h$ plane. Assuming $\rho_{p}=0$, Equation (5) represents an ellipse with the semiaxes $\rho_{k}$ and $\rho_{k} / \sin \theta$. The area of the ellipse is $\pi \rho_{k}^{2} / \sin \theta$, and the variation area of $d$ and $h$ for the above-mentioned 13 planets is $18.22 \times 0.1=1.822$. Hence, of these 13 planets the number that can collide with the comet is

$$
\frac{\pi \rho_{k}^{2} \times 13}{2 \sin \theta \times 1.822} \ll 1
$$

But the 13 minor planets correspond to 1735 discovered planets, and to each discovered planet there correspond

$$
\frac{1}{1735} \int_{m_{p}}^{m_{k}} \frac{2.3 \times 10^{-14}}{m^{2.28}} \mathrm{~d} m
$$


undiscovered ones. Hence, the total number of collisions per $n$ revolutions of the comet will be

$$
n \frac{\pi p_{k}^{2} \times 13}{2 \sin \theta \times 1.822} \frac{1}{1735} \int_{m_{p}}^{m_{k}} \frac{2.3 \times 10^{-14}}{m^{2.28}} \mathrm{~d} m .
$$

It follows that during 100 revolutions there is a good chance of a collision with a microplanet of mass $4.05 \times 10^{8} \mathrm{~g}$. The lower limit of the integral is of great importance since the number of planets grows rapidly as the mass decreases, and it is therefore assumed in our calculations that there is a collision with a planet whose mass is equal to the lower limit. About 60 short-period comets of the Jupiter family have been discovered so far. This means that it is possible for one of them to meet a larger microplanet; and during 100 revolutions one of them may meet a microplanet of mass $9.9 \times 10^{9} \mathrm{~g}$ and radius $8.6 \mathrm{~m}$. So far we have considered microplanets whose masses are greater than $10^{8} \mathrm{~g}$ (or $5 \times 10^{-26}$ solar masses). If we admit microplanets of mass $10^{7} \mathrm{~g}$, the number of collisions per revolution will become about 3.5 times greater, and a single comet may collide with an object of mass $1.2 \times 10^{7} \mathrm{~g}$ and radius $90 \mathrm{~cm}$ in a single revolution.

\section{Perturbations in $\mathbf{1} / a$}

When estimating maximum perturbations (without collisions) it is possible to make use of H. A. Newton's formula

$$
\Delta \frac{1}{a}=-\frac{4 m_{p}}{S^{2}} \frac{h \sin ^{2} \theta}{d^{2}+h^{2} \sin ^{2} \theta} \text {. }
$$

Let us determine the mean value $M[\Delta(1 / a)]$ of the values of $\Delta(1 / a)$ that arise when the minor planets come sufficiently close to the comet that $d$ and $h$ assume all possible values over the intervals $\left(0, d_{0}\right)$ and $\left(-h_{0}, h_{0}\right)$. No collisions will occur in an ellipse around the centre $d_{0}=0, h_{0}=0$, and we call this region $Q_{1}$. From symmetry it follows that

$$
M[\Delta(1 / a)]=0 .
$$

For determining the dispersion we exclude the semicircle near the centre of the $d, h \sin \theta$ plane that has a radius smaller than that of the smallest planet, i.e., $p_{0}=$ $\left(d^{2}+h^{2} \sin ^{2} \theta\right)^{1 / 2} \approx 10^{-9}$. This will not cause an appreciable error since there are no collisions with minor planets more massive than the comet. In this case the dispersion is determined according to the formula

$$
\begin{aligned}
D\left(\Delta \frac{1}{a}\right) & =\iiint_{Q}\left(\Delta \frac{1}{a}\right)^{2} p(N) \mathrm{d} d \mathrm{~d} h \mathrm{~d} m_{p} \\
& =\frac{1.28\left(m_{0} M_{0}\right)^{1.28}}{M_{0}^{1.28}-m_{0}^{1.28}} \frac{16}{S^{2}} \iint_{Q_{1}} \frac{h^{2} \sin ^{4} \theta}{\left(d^{2}+h^{2} \sin ^{2} \theta\right)^{2}} \mathrm{~d} d \mathrm{~d} h \int_{m_{0}}^{M_{0}} \frac{m_{p}^{2}}{m_{p}^{2.28}} d m_{p} \\
& \simeq \frac{95\left(m_{0} M_{0}\right)^{1.28}}{M_{0}^{1.28}} \frac{\left(M_{0}^{0.72}-m_{0}^{0.72}\right)}{-m_{0}^{1.28}} \iint_{Q_{1}} \frac{h^{2} \sin ^{4} \theta}{\left(d^{2}+h^{2} \sin ^{2} \theta\right)^{2}} \mathrm{~d} d \mathrm{~d} h
\end{aligned}
$$


Introducing the polar coordinates

we have

$$
d=p \cos \varphi, \quad h \sin \theta=p \sin \varphi,
$$

$$
D\left(\Delta \frac{1}{a}\right)=95 \frac{\left(m_{0} M_{0}\right)^{1.28}\left(M_{0}^{0.72}-m_{0}^{0.72}\right)}{M_{0}^{1.28}-m_{0}^{1.28}} \int_{0 p_{0}}^{\pi p_{1}} \frac{\sin \theta p^{2} \sin ^{2} \varphi}{p^{4}} p \mathrm{~d} \varphi \mathrm{d} p .
$$

Since $m_{0} \ll M_{0}$ and $\left|\ln p_{1}\right| \gg\left|\ln p_{0}\right|$ we have

$$
D\left(\Delta \frac{1}{a}\right) \approx 150 \sin \theta \ln p_{1} M_{0}^{0.72} m_{0}^{1.28} \text {. }
$$

It is obvious that in our case $D[\Delta(1 / a)]<10^{-28}$. When we modelled this process by the Monte Carlo method on an electronic computer we obtained $D[\Delta(1 / a)]<10^{-29}$.

Let us apply now Kolmogorov's inequality, which is that if mutually independent random values $\zeta_{1}, \zeta_{2}, \ldots, \zeta_{n}$ have finite dispersions, then the probability that

$$
\left|\sum_{s=1}^{k} \zeta_{s}-M \zeta_{s}\right|<\varepsilon \quad(k=1, \ldots, m)
$$

is not less than

$$
1-\frac{1}{\varepsilon^{2}} \sum_{k=1}^{m} D \zeta_{k}
$$

In our case $M \zeta_{k}=0$, all the $D \zeta_{k}$ are equal, and $m$ is the number of minor planets coming into the contour of radius $p_{1}$. The probability that the sum of the increments $\Delta(1 / a)$ over 100 revolutions of the comet will not exceed $10^{-8}$ at any point, i.e.,

$$
\left|\sum_{k=1}^{m} \zeta_{k}\right|<10^{-8}
$$

will thus not be less than

$$
1-10^{16} \sum_{k=1}^{m} D \zeta_{k} \text {. }
$$

From Equation (1), the number of minor planets $m$ having $d$ in the interval $(0.0,0.1)$ is

$$
m=\frac{13}{1735} \int_{m_{p}}^{m_{k}} \frac{2.3 \times 10^{-14}}{m^{2.28}} \mathrm{~d} m \approx 10^{8} .
$$

Then, substituting in Equation (8), we have

$$
1-10^{16} \sum_{k=1}^{m} D \zeta_{k}>1-10^{-4} \text {. }
$$

\section{References}

Belyaev, N. A.: 1966, Byull. Inst. Teor. Astron. 10, 696.

Chebotarev, G. A.: 1968, Efemeridy Malykh Planet na 1969 God, Nauka, Leningrad.

Kuiper, G. P., Fujita Y., Gehrels, T., Groeneveld I., Kent, J., Van Biesbroeck, G., and van Houten, C. J.: 1958, Astrophys. J. Suppl. 32, 289.

van Woerkom, A.: 1948, Bull. Astron. Inst. Neth. 10, 445. 